\title{
Potassium diperiodatocuprate-mediated preparation of poly(methyl methacrylate)/organo-montmorillonite composites via in situ grafting copolymerization
}

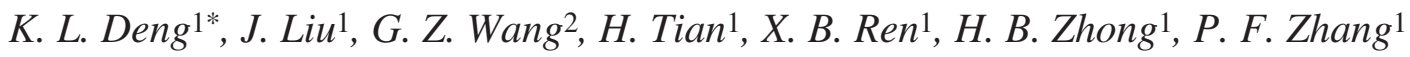 \\ ${ }^{1}$ College of Chemistry and Environmental Science, Hebei University, Baoding, 071002, China \\ 2Zunhua Public Bureau Hygienic Supervision Institute, Zunhua, 064200, China
}

Received 29 June 2008; accepted in revised form 17 August 2008

\begin{abstract}
In this study, potassium diperiodatocuprate $\left(\mathrm{Cu}^{3+}\right)$ was selected as an initiator to prepare poly(methyl methacrylate)/organo-montmorillonite composites (OMMT- $g$-PMMA) by in situ graft copolymerization. Three synthetic parameters were systematically evaluated as a function of the temperature, the concentration of initiator, $\mathrm{pH}$ and the ratio of MMA to OMMT. It was found that $\mathrm{Cu}^{3+}$ was a highly efficient initiator for the preparation of OMMT- $g$-PMMA i.e., monomer conversion and grafting efficiency were as higher as $95 \%$. The X-ray diffraction measurement showed the intercalation of PMMA chains into OMMT layers on base of an increasing basal spacing after polymerization. FTIR analysis also suggested that the PMMA chains were effectively grafted onto OMMT substrate. The enhanced thermal stabilities of OMMT$g$-PMMA composites were confirmed by the thermal gravimetric analysis (TGA). Finally, a single-electron-transfer mechanism was proposed to illustrate the formation of radicals and the preparation process of OMMT- $g$-PMMA composites. $\mathrm{Cu}^{3+}$ can be used as an effective and practical initiator in preparing the organic/inorganic composite due to its high grafting efficiency and the milder reaction condition.
\end{abstract}

Keywords: polymer composites, potassium diperiodatocuprate, montmorillonite

\section{Introduction}

Organic/inorganic nanocomposite, as a special type of material, has attracted considerable interests in the recent years [1-3]. The nanocomposites were actually obtained by the successful incorporation of an organic polymer and an inorganic component into one single material. Various minerals $[4,5]$, metal particles [6], and metal oxides [7] are often used as the inorganic component in the composites and the organic polymer includes polystyrene [8], polymethyl methacrylate [9], polypropylene [10], and so on. The organic/inorganic composites usually differ from both the original pure polymers and the inorganic particles in some chemical and physical properties. In comparison with the single com- ponent, the composites often show many unique appealing properties such as enhanced mechanical property [11], fire retardance and excellent thermal stability $[12,13]$, good ionic conductivity [14] and gas barrier property [15]. For example, the elastic modulus of the nano-composite composed of polyester and 5\% nanoclay was increased to 6646 from $5393 \mathrm{MPa}$ for unreinforced polyester [11]. The enhancement in ion conductivity of polymethyl methacrylate $/ \mathrm{CeO}_{2}$ was observed due to the increase in the number of charge carriers by reaction of $\mathrm{CeO}_{2}$ with anion and cation [14].

To date, several synthetic procedures have been developed in order to prepare the composites. For instance, the typical synthetic methods used to pro-

*Corresponding author, e-mail: dk1369@hbu.edu.cn

(C) BME-PT and GTE 
duce polymer/mineral clay nanocomposites are in situ intercalative polymerization [16], exfoliationadsorption [17], melt intercalation [18] and template synthesis etc. [19]. It is well known that the grafting reaction of monomers onto some inorganic particles is also an effective strategy to prepare the corresponding organic/inorganic composites [2023]. In the preparation of these composites, the polymers chains grow from inorganic material substrates by the grafting reaction. Besides, the terminal reactive groups on the polymer chains can directly react with the reactive ones bound to the substrates. Luo et al. described the preparation of a novel nano-superabsorbent composite by graft copolymerization of acrylamide and acrylic acid onto montmorillonite/starch initiated using ${ }^{60} \mathrm{Co}-$ ray irradiation [20]. The polymer/silica composite was synthesized via the grafting copolymerization of the polymers having pendant peroxycarbonate groups and the silica with azo groups in the presence of vinyl acetate [22].

In our previous work [24-28], many graft copolymerizations initiated by the high valence metal ions of the common monomers, especially onto the synthetic and natural polymers have been investigated widely. The high valence transition metals ions, including $\mathrm{Ni}^{4+}, \mathrm{Ag}^{3+}$ and $\mathrm{Cu}^{3+}$ ions, have proved to be the highly efficient initiators. For instance, potassium diperiodatocuprate was successfully employed to initiate the graft copolymerization of methyl acrylate onto chitosan. On the optimal reaction condition, the grafting efficiency reached as high as 95\% [24]. Also, the graft copolymer with high graft parameters from methyl acrylate and poly(vinyl alcohol) was prepared using potassium diperiodatonickelate $\left(\mathrm{Ni}^{4+}\right)$ or potassium diperiodatoargentate $\left(\mathrm{Ag}^{3+}\right)$ as the initiators $[25,28]$. In the aforementioned studies on the grafting copolymerization, the radicals formed by the redox reaction of the high valence metal ions with some reductive groups on the substrate. Actually, the redox reaction of the metal ions underwent a two-step single electron-transfer progress. This radical-producing mechanism has been used to explain the formation of the graft sites and the initiation in the graft copolymerization [24-26]. If the high valence metal ions are used to initiate the graft copolymerization of the common monomers onto some inorganic materials such as glass fiber, montmoril- lonite, and kaolin, a new method for the preparation of the corresponding composites can be developed. Poly (methyl methacrylate) (PMMA) and montmorillonite were selected as the two organic/ inorganic components in the composite prepared in this study. PMMA is an amorphous, transparent polymer and widely used in various fields. On the other hand, montmorillonite is often selected as the inorganic component in the preparation of organic/ inorganic composite. Because of the hydrophobic nature of montmorillonite, it is often modified by exchanging of an alkyl ammonium ions with the cations between the silicate layers. As an extension of our previous work, the high valence metal ions were applied to the inorganic material field in this study, that is, potassium diperiodatocuprate $\left(\mathrm{Cu}^{3+}\right)$ was used as an initiator for the preparation of PMMA/OMMT composite via the in situ grafting copolymerization. The synthetic parameters for composites were evaluated by varying the temperature, monomer concentration, and initiator concentration. The experimental result has shown that potassium diperiodatocuprate is an efficient initiator for the synthesis of OMMT- $g$-PMMA composite via the grafting copolymerization. Theoretically speaking, the titled OMMT-g-PMMA composite can be directly used as an inorganic clay-modified material or a new potential blend-compatilizer in the modification of organic polymers with inorganic minerals.

\section{Experimental section}

\subsection{Materials}

Organophilic montmorillonite (OMMT) (DK-2) used in this study was purchased from Fenghong Clay Chemicals Co. Ltd., Zhejiang Province, and received as fine particle powders. The surfactant used to modify montmorillonite was a long-chain organic amine salt. Methyl methacrylate (MMA) was washed successively with aqueous sodium hydroxide and distilled water to remove the hydroquinone inhibitors, and then it was dried over anhydrous sodium sulfate overnight and finally distilled. The grafting initiator, potassium diperiodatocuprate $\left(\mathrm{Cu}^{3+}\right)$ was synthesized and characterized according to the reported literature [24, 29]. The other chemicals were analytical grade and were used without any further purification. 


\subsection{Measurements}

The structures of OMMT- $g$-PMMA, OMMT and pure PMMA were characterized by Fourier transform infrared spectrum with an FTS-40 spectrophotometer (BIORAD, USA) in potassium bromide pellets. The X-ray diffraction pattern was measured with a Rigaku D/MAX 2400 X-ray diffractometer. The $\mathrm{X}$-ray beam was derived from nickel-filtered $\operatorname{CuK}_{\alpha}(\lambda=0.154 \mathrm{~nm})$ radiation in a sealed tube operated at $40 \mathrm{kV}$ and $200 \mathrm{~mA}$. The experiments were conducted in the angle range $1-50^{\circ}$, at a scanning rate of $8 \% \mathrm{~min}$ and a scanning step of $0.02^{\circ}$. The thermo-gravimetric analysis (TGA) curves were completed with a Shimadzu DGC-40 DTATG apparatus (Shimadzu, Japan) in $\mathrm{N}_{2}$ atmosphere at a heating rate of $10^{\circ} \mathrm{C} / \mathrm{min}$.

\subsection{Preparation of OMMT-g-PMMA composite and treatment}

A series of the graft copolymerizations were carried out in a glass tube $(20 \mathrm{~mm}$ in diameter and $200 \mathrm{~mm}$ in length) equipped with a magnetic bar. In the typical procedure, $0.5 \mathrm{~g}$ OMMT, deionized water and required amount of MMA were added into a tube. Then, several standard cycles of evacuation and backfill with dry and pure nitrogen was performed to thoroughly remove oxygen. The tube was charged with the oxygen-free $\mathrm{Cu}^{3+}$ aqueous solution and water-acetone by a syringe. The total volume of reaction system was controlled at $6 \mathrm{ml}$ by the addition of mixed solvent (water/acetone $=$ $3: 1$ ). Herein, it is necessary to state that the aim of addition of some acetone is to maintain more uniform dispersion of OMMT during the graft copolymerization. The graft copolymerization was performed under the designated conditions of different monomer concentrations, initiator concentrations, $\mathrm{pH}$ and temperatures. After a definite time, the polymerization was terminated by several drops of hydrochloric acid solution. Subsequently, the reaction mixture was poured into a large amount of methanol, and the crude graft copolymer was filtered through a weighted, sintered glass funnel. The crude graft copolymer was dried at $90^{\circ} \mathrm{C}$ in air for several hours and then dried to a constant weight under vacuum at $60^{\circ} \mathrm{C}$. The exhaustive Soxhlet extraction with acetone for $48 \mathrm{~h}$ was performed to remove some homopolymers in the crude graft copolymer. The resulting OMMT- $g$-PMMA com- posite was dried at $60^{\circ} \mathrm{C}$ to a constant weight under vacuum.

Monomer conversion $(C \%)$, grafting percentage $(P \%)$ and grafting efficiency $(E \%)$ were designated as the synthetic parameters in the preparing OMMT- $g$-PMMA composites, and were defined in the following manner (see Equations (1)-(3)):

$C \%=($ total weight of PMMA $\div$ weight of MMA charged) $100 \%$

$P \%=($ weight of PMMA grafted $\div$ weight of substrate) $\cdot 100 \%$

$E \%=($ weight of PMMA grafted $\div$ total weight of PMMA formed) $\cdot 100 \%$

\section{Results and discussion}

\subsection{Effect of the different factors on synthetic parameters}

\subsubsection{Effect of $\mathrm{Cu}^{3+}$ concentration}

The effect of $\mathrm{Cu}^{3+}$ concentration on synthetic parameters was investigated when the other reaction conditions are kept unchanged. As shown in Figure 1, the three synthetic parameters exhibited a considerable dependence on the $\mathrm{Cu}^{3+}$ concentration, especially for $\mathrm{C} \%$ and $\mathrm{P} \%$. With increasing $\mathrm{Cu}^{3+}$ concentration in the range of $0.6-$ $0.8 \cdot 10^{-3} \mathrm{~mol} / \mathrm{l}, \mathrm{C} \%, P \%$, and $E \%$ all increased quickly. For example, when the $\mathrm{Cu}^{3+}$ concentration was $0.65 \cdot 10^{-3}$ and $0.80 \cdot 10^{-3} \mathrm{~mol} / 1, C \%$ and $P \%$ reached to 79.9 from $46.2 \%$, and 508 from $222 \%$, respectively. However, beyond the $\mathrm{Cu}^{3+}$ concentration of $0.8 \cdot 10^{-3} \mathrm{~mol} / \mathrm{l}, \mathrm{C} \%$, and $P \%$ are found to

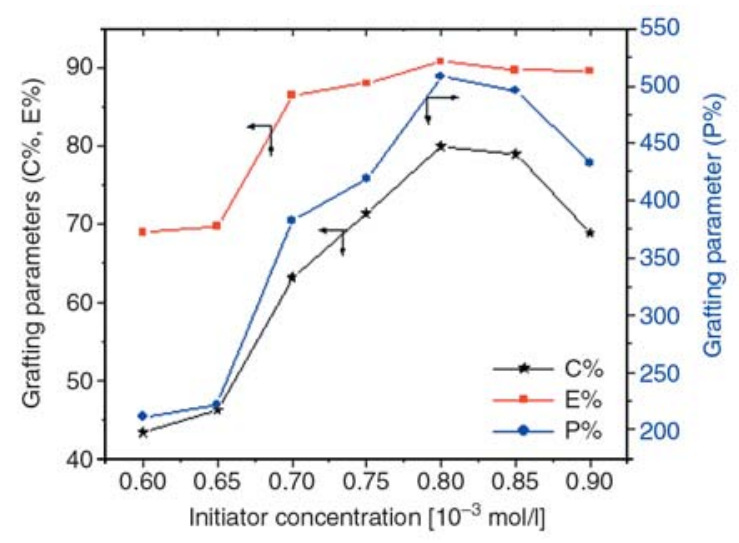

Figure 1. Effect of initiator concentration on synthetic parameters (time $=3 \mathrm{~h}$; temperature $=25^{\circ} \mathrm{C}$; MMA/OMMT $=8 ; \mathrm{pH}=11.7$ ) 
decrease. In another word, the optimum value of $\mathrm{Cu}^{3+}$ concentration was $0.8 \cdot 10^{-3} \mathrm{~mol} / \mathrm{l}$ in this study. In the lower concentration of $\mathrm{Cu}^{3+}$ ranging $0.6-$ $0.8 \cdot 10^{-3} \mathrm{~mol} / \mathrm{l}$, the higher $\mathrm{Cu}^{3+}$ concentration in the reaction system led to increasing in the number of macro-radicals formed by redox reaction of $\mathrm{Cu}^{3+}$. Therefore, $C \%, P \%$ and $E \%$ increased as $\mathrm{Cu}^{3+}$ concentration grew up. A similar observation was also found in the $\mathrm{Cu}^{3+}$-initiated graft copolymerization of acrylic acid [30]. If, however, excessive $\mathrm{Cu}^{3+}$ ions were added into the reaction system, the probability of the collision between $\mathrm{Cu}^{3+}$ ions with the radicals formed was greatly enhanced. As a result, the partial radicals produced in the reaction were again converted into some inert species by the excessive $\mathrm{Cu}^{3+}$ ions [24]. This side-reaction likely decreased the number of grafting radicals formed in the system and also terminated the continuous propagation of some copolymer chains. It is necessary to mention that compared with $C \%$ and $P \%$, $E \%$ was basically independent on the higher $\mathrm{Cu}^{3+}$ concentration range. This experimental result indicated the homo-polymerization of MMA was not increased with $\mathrm{Cu}^{3+}$ concentration.

In order to testify the effective grafting copolymerization initiated by $\mathrm{Cu}^{3+}$ ions, the comparative experiments have been conducted. When potassium persulfate ( $2 \%$ to monomer) rather than $\mathrm{Cu}^{3+}$ was added into the tube with required amount of MMA, acetone/water, and OMMT at $45^{\circ} \mathrm{C}$, no obvious polymer was obtained after $2 \mathrm{~h}$. At the same time, no polymerization behaviors were observed in the OMMT-free reaction system using $\mathrm{Cu}^{3+}$ as initiators. If the above-mentioned experiment contained OMMT and MMA was carried out at $45^{\circ} \mathrm{C}$ using $\mathrm{Cu}^{3+}$ as an initiator, a large amount of polymers were obtained after $2 \mathrm{~h}$. This phenomenon implied that $\mathrm{Cu}^{3+}$ ion acted as an effective redox initiator for the polymerization of MMA at lower temperature. The radicals were easily formed through the redox reaction of $\mathrm{Cu}^{3+}$ ion with some reductive groups on OMMT. For potassium persulfate, no obvious decomposition takes place at $45^{\circ} \mathrm{C}$ to produce the corresponding radicals. The characteristics of the formation of radicals via the $\mathrm{Cu}^{3+}$ redox reaction have been reported in several publications [29-31].

\subsubsection{Effect of the ratio of MMA to OMMT}

The effect of MMA/OMMT ratio on the three synthetic parameters is depicted in Figure 2. With increasing the ratio of MMA to OMMT, $P \%, E \%$ and $C \%$ increase drastically. For instance, as the ratio of MMA to OMMT was fixed at 5 and 7, C\% reached $47.7,83.7 \%$, and $P \%$ were $189,480 \%$, respectively. Apparently, the concentration of MMA was heightened with increasing the ratio of MMA/OMMT because the total volume of reaction system and OMMT was kept unchanged. In addition, the number of radical sites on OMMT could be considered as constant when other reaction conditions were kept invariable. According to the basic principle of radical polymerization, $\mathrm{C} \%$ and $\mathrm{P} \%$ affirmatively augmented with an increasing of MMA/OMMT ratio. The higher concentration of MMA in close vicinity to each radical site resulted in the quicker polymerization rate and the production of more polymers in the reaction.

Additionally, three synthetic parameters showed remarkable declining tendency when the ratio of MMA/OMMT was larger than 8. As the ratio of MMA/OMMT was 8 and $10, C \%$ decreased to 18.9 from $84.7 \%$; $P \%$ went down to 85 from $623 \%$, respectively. The higher ratio of MMA/OMMT implied that the less water was added into the system because of the fixed volume in the polymerization. Furthermore, the graft copolymerization was carried out in heterogeneous systems because $\mathrm{Cu}^{3+}$ dissolves only in water, MMA, however, dissolves in some organic solvents, and OMMT is not soluble in common solvent. As a result, the collision probability between OMMT and $\mathrm{Cu}^{3+}$ was greatly decreased at the higher ratio of MMA/OMMT. The

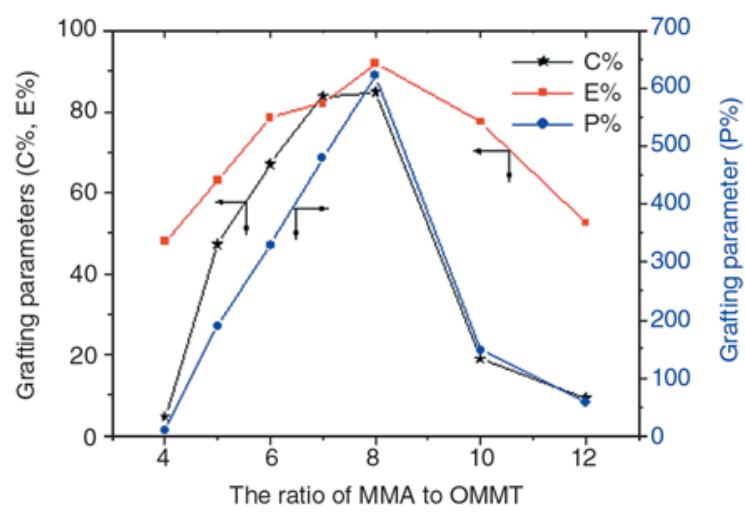

Figure 2. Effect of the ratio of MMA to OMMT on synthetic parameters $($ time $=3 \mathrm{~h}$; temperature $=$ $25^{\circ} \mathrm{C} ;\left[\mathrm{Cu}^{3+}\right]=0.8 \cdot 10^{-3} \mathrm{~mol} / \mathrm{l} ; \mathrm{pH}=11.7$ ) 
number of radicals was decreased with increasing ratio of MMA/OMMT, finally resulting in a considerable fall in the synthetic parameters.

\subsubsection{Effect of temperature}

The effect of reaction temperature on synthetic parameters has been studied by varying the temperature in the range of $0-65^{\circ} \mathrm{C}$. As shown in Figure 3, it is found that $E \%$ is slightly dependent on the reaction temperature between 25 and $50^{\circ} \mathrm{C}$. On the other hand, $\mathrm{C} \%$ and $P \%$ exhibited a remarkable dependence on the temperature selected in graft copolymerization. The activation energy was a small positive value when investigating the redox reaction of $\mathrm{Cu}^{3+}$ ions with some reducing groups including amino and hydroxyl groups [29, 32]. Furthermore, the radicals were produced via a single electron transfer process in $\mathrm{Cu}^{3+}$ redox reaction and initiated the polymerization of acrylonitrile [29, 32]. So, the formation rate of radicals is accelerated with increasing temperature, leading to the increase of $\mathrm{C} \%$ and $\mathrm{P} \%$. For example, when the reaction temperature was controlled at 12 and $25^{\circ} \mathrm{C}, \mathrm{C} \%$ increased to 94.5 from $35.1 \%$ and $\mathrm{P} \%$ climbed up to 580 from $229 \%$, respectively.

In addition, $C \%$ and $P \%$ decreased drastically as the temperature was further increased to 65 from $25^{\circ} \mathrm{C}$. The reason for this phenomenon is mainly ascribed to the following fact. Many radicals were formed via the redox reaction of $\mathrm{Cu}^{3+}$ ions within a short time period at higher temperature. The graft copolymerization was conducted in the heterogeneous system because of the different solubilities of MMA, OMMT, and $\mathrm{Cu}^{3+}$ ions. It took a certain

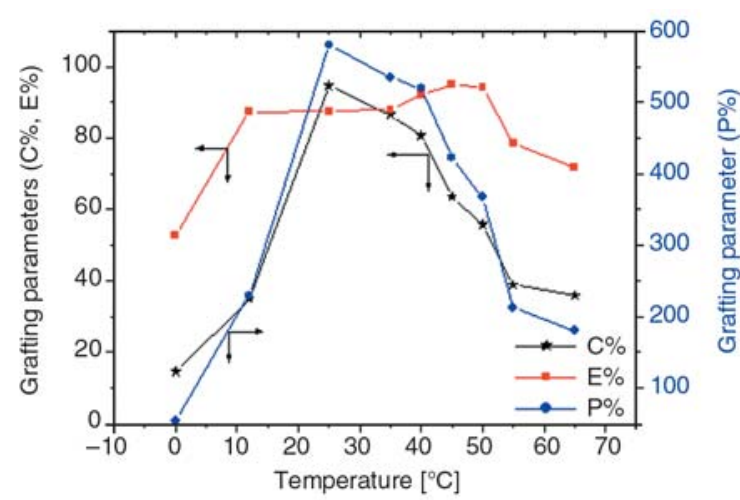

Figure 3. Effect of temperature on synthetic parameters (time $=3 \mathrm{~h} ;\left[\mathrm{Cu}^{3+}\right]=0.8 \cdot 10^{-3} \mathrm{~mol} / \mathrm{l}$; MMA/OMMT $=8 ; \mathrm{pH}=11.7$ ) time for the MMA to approach the formed radicals via diffusion and subsequently to polymerize. In this case, many radicals were likely scavenged through the bimolecular termination or other byreactions before the radicals initiated the polymerization of MMA. Consequently, $\mathrm{C} \%$ and $\mathrm{P} \%$ exhibited a decreasing tendency with increasing the temperature. At the higher temperature, the chain transfer reaction of the radicals to the molecules except OMMT was also enhanced owing to the increased activity of the radicals. Therefore, the synthetic parameter $E \%$ showed decreasing trend to some extent especially over $50^{\circ} \mathrm{C}$. Savina et al. presented the similar results when investigating the effect of temperature on the grafting parameters in graft copolymerization [30]. By the overall comparison among three synthetic parameters, the other experiment was carried out at $25^{\circ} \mathrm{C}$ to obtain the higher synthetic parameters.

\subsubsection{Effect of $\mathrm{pH}$}

Figure 4 has shown the effect of $\mathrm{pH}$ value on the synthetic parameters. In this study, the hydrochloric acid or potassium hydroxide solution were used as $\mathrm{pH}$-adjustor to control the required alkalinity in the reaction system. The synthetic parameters were evaluated under the different $\mathrm{pH}$ value in the range of 8.8-13.0. It was found that $C \%, E \%$ and $P \%$ were significantly dependent on $\mathrm{pH}$ value in the reaction system. The three synthetic parameters increased drastically with $\mathrm{pH}$ value, and thereafter went down as the $\mathrm{pH}$ value further increased. $C \%$ and $P \%$ went up to the highest value (74.3 and $475 \%$ ) at $\mathrm{pH}=11.7$, whereas the graft parameter

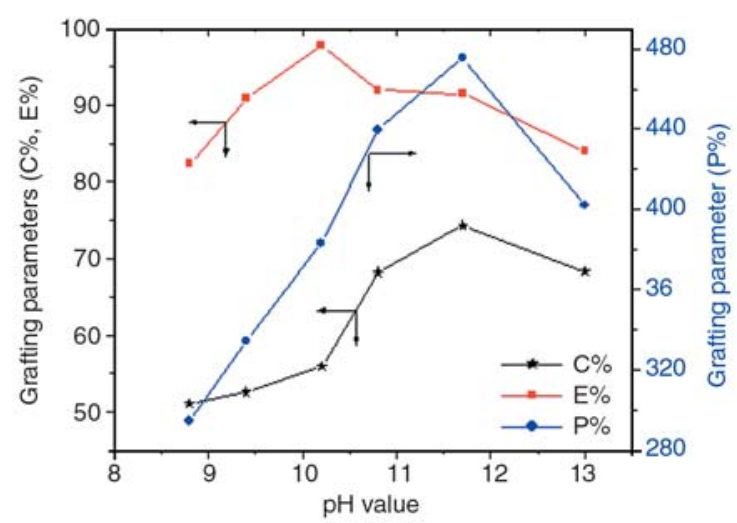

Figure 4. Effect of $\mathrm{pH}$ on synthetic parameters (time $=3 \mathrm{~h}$; temperature $=25^{\circ} \mathrm{C}$; $\left.\left[\mathrm{Cu}^{3+}\right]=0.8 \cdot 10^{-3} \mathrm{~mol} / \mathrm{l} ; \mathrm{MMA} / \mathrm{OMMT}=8\right)$ 
$E \%$ reached its highest value at $\mathrm{pH}=10.2$. The results indicated the presence of optimal $\mathrm{pH}$ value in the preparation of the composites.

The two main active forms of $\mathrm{Cu}^{3+}$ complexes including $\left[\mathrm{Cu}\left(\mathrm{H}_{3} \mathrm{IO}_{6}\right)_{2}(\mathrm{OH})_{2}\right]^{3-}$ and $\left[\mathrm{Cu}\left(\mathrm{H}_{2} \mathrm{IO}_{6}\right)\right.$ $\left.\left(\mathrm{H}_{3} \mathrm{IO}_{6}\right)\right]^{2-}$ have been detected in alkaline medium. $\mathrm{Cu}^{3+}$ complex species actually vary with the alkalinity in the medium, because the dissociation of the various species of $\mathrm{H}_{5} \mathrm{IO}_{6}$ obviously depends on the $\mathrm{pH}$ value $[29,32]$. Most importantly, the different forms of $\mathrm{Cu}^{3+}$ complexes have different reactivity in the redox reaction. At excessively low or high pH range, the $\mathrm{H}_{3} \mathrm{IO}_{6}^{2-}$ and $\mathrm{H}_{2} \mathrm{IO}_{6}^{3-}$ ions all decrease, which further induces to decrease the concentration of active $\left[\mathrm{Cu}\left(\mathrm{H}_{3} \mathrm{IO}_{6}\right)_{2}(\mathrm{OH})_{2}\right]^{3-}$ and $\left[\mathrm{Cu}\left(\mathrm{H}_{2} \mathrm{IO}_{6}\right)\right.$ $\left.\left(\mathrm{H}_{3} \mathrm{IO}_{6}\right)\right]^{2-}$ species. Thus, $\mathrm{pH}$ value plays a key role in the formation of radicals in the $\mathrm{Cu}^{3+}$ redox reaction, and determines the number of radicals used for the polymerization. By the estimation based on the dissociation constants of periodic acid [32], the $\mathrm{pH}$ value at which the higher synthetic parameters were obtained is basically close to that for the existence of $\mathrm{H}_{3} \mathrm{IO}_{6}^{2-}$ and $\mathrm{H}_{2} \mathrm{IO}_{6}^{3-}$ ions. The same effecting behaviors of $\mathrm{pH}$ on the grafting parameters were also observed in the other $\mathrm{Cu}^{3+}$-initiated grafting system [30]. Because the property of composites mainly depends on $P \%$ rather than $E \%$, we selected $\mathrm{pH}=11.7$ to carry out other experiments.

\subsection{Characterization of the OMMT- $g$-PMMA composites}

\subsubsection{FTIR measurements}

In order to illustrate the structural change before and after polymerization, the FTIR spectra of original OMMT, the pure PMMA, and OMMT- $g$ PMMA composite $(P \%=600 \%)$ were recorded in the range of $4000-400 \mathrm{~cm}^{-1}$. As shown in Figure 5, the two peaks at about 3600 and $3450 \mathrm{~cm}^{-1}$ in the spectrum of OMMT implied the presence of the reactive groups including $-\mathrm{OH}$ and $-\mathrm{NH}-$. In the FTIR spectrum of the OMMT- $g$-PMMA composite, the strong absorption bands at $1731.8 \mathrm{~cm}^{-1}$ was observed, corresponding to the characteristics of $\mathrm{C}=\mathrm{O}$ stretching vibration from PMMA. Also, the characteristic absorption bands at 1149.2, 1192, and $1243.2 \mathrm{~cm}^{-1}$ could be ascribed to the $\mathrm{C}-\mathrm{O}-\mathrm{C}$ stretching vibration in PMMA chains [21]. Furthermore, several characteristic absorption peaks attributed to OMMT were also recorded in the FTIR

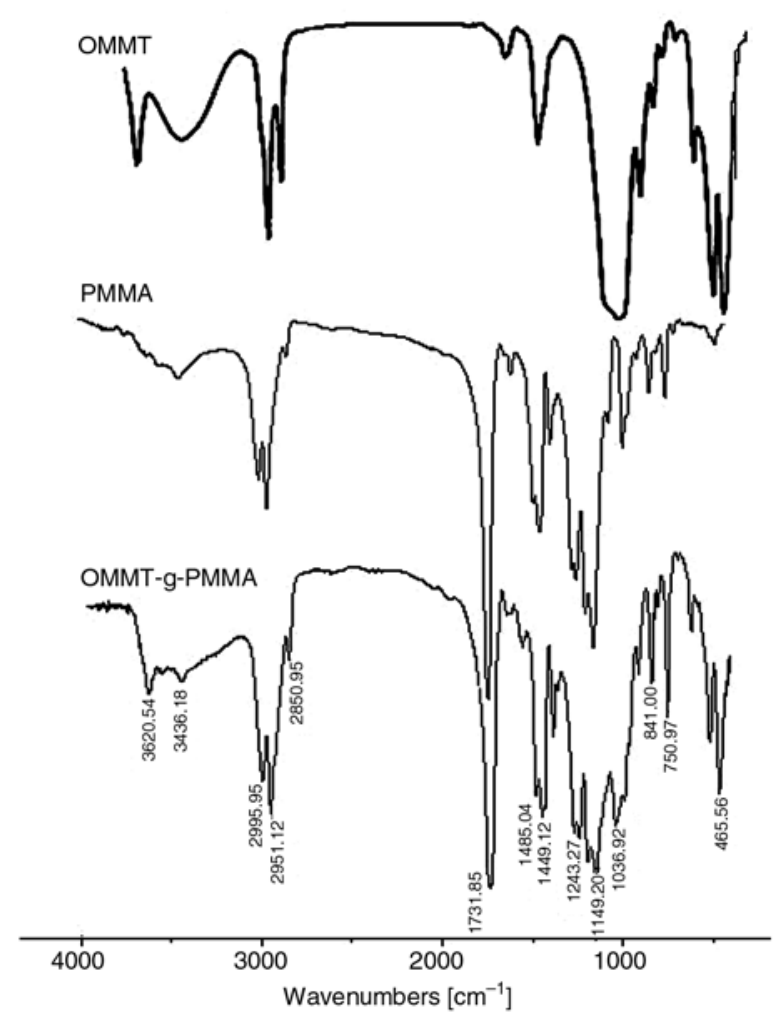

Figure 5. FTIR spectra of OMMT, PMMA and OMMT- $g$ PMMA composites

spectrum of the composite. For instance, the absorption peaks at $1036.9 \mathrm{~cm}^{-1}$ corresponded to the $\mathrm{Si}-\mathrm{O}-\mathrm{Si}$ stretching vibration from OMMT substrate. The bands at 3620.5 and $3436.1 \mathrm{~cm}^{-1}$ were related to the reactive groups in OMMT. From these spectral data, it could be concluded that the composite was consisted of both PMMA and OMMT moieties. Therefore, the PMMA chains were chemically grafted onto OMMT because the composite has been completely extracted with a large amount acetone.

\subsubsection{TGA analysis}

The thermo-gravimetric analysis (TGA) of OMMT, PMMA and OMMT- $g$-PMMA $(P \%=$ $550 \%$ ) has illustrated the decomposition behaviors under heating in an inert atmosphere. As shown in Figure 6, the weight loss behavior regarding water likely bound to the OMMT- $g$-PMMA was observed in the range of $100-260^{\circ} \mathrm{C}$. The second step degradation of OMMT- $g$-PMA composite started at about $275^{\circ} \mathrm{C}$, and the final decomposition temperature was found to be $405^{\circ} \mathrm{C}$. This process mainly corresponded to the decomposition of the 


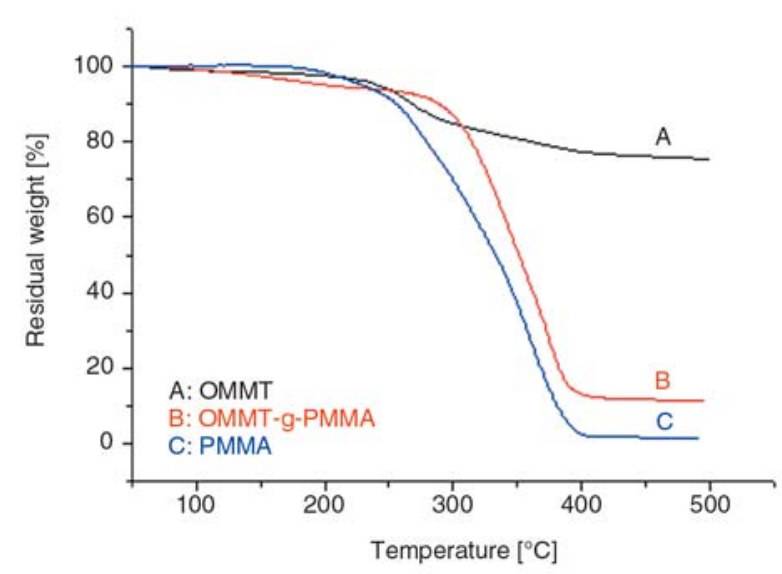

Figure 6. Thermal analysis for OMMT, OMMT- $g$-PMMA and PMMA

organic component in the composite. On the other hand, the pure PMMA exhibited only one step degradation between $180-400^{\circ} \mathrm{C}$. Compared with the pure PMMA, the initial decomposition temperature of OMMT- $g$-PMMA was about $95^{\circ} \mathrm{C}$ higher than that of pure PMMA. The improvement in the thermal stability for OMMT-g-PMMA is ascribed to the introduction of OMMT into PMMA. The inorganic clay component effectively absorbed or retarded the heat in the OMMT-g-PMMA thermal degradation. In addition, the finally residual weight of OMMT- $g$-PMMA was found to be around $11.8 \%$. This value is in a good agreement with the one calculated from the organic-component content in OMMT and the grafting percentage of sample used.

\subsubsection{XRD patterns}

As shown in Figure 7, the X-ray diffraction patterns of OMMT, OMMT- $g$-PMMA and the mixture of PMMA and OMMT were measured to investi-

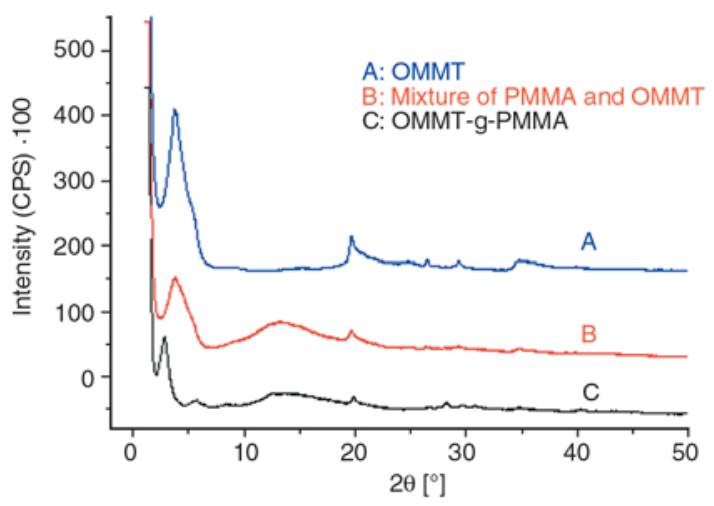

Figure 7. X-ray diffraction patterns of OMMT, OMMT- $g$ PMMA and the mixture of PMMA/OMMT gate the change of OMMT structure after grafting. In the XRD pattern of pure OMMT, several diffraction peaks at $2 \theta=3.68,19.62$, and $34.78^{\circ}$, were recorded, which implied the presence of the ordered layer-like structure of OMMT [2]. The basal spacing corresponding to the $2 \theta=3.68^{\circ}$ was $2.40 \mathrm{~nm}$ according to Bragg's equation. In order to further elucidate the influence of polymerization on the OMMT structure, the XRD of a mixture of the pure PMMA and OMMT was also determined at the same $2 \theta$ range. Obviously, except for a broad diffraction peak ascribed to PMMA at $2 \theta$ from about 8 to $20^{\circ}$, the XRD pattern of the mixture was almost the same as that of pure OMMT substrate. This result suggested the layer-like structure of OMMT did not change after the simple mixing.

In the small angle region of the XRD pattern for the composite, however, three sharp diffraction peaks at $2.74,5.56$ and $8.32^{\circ}$ were recorded, indicating an ordered structure. Considerably, the diffraction peak at $3.68^{\circ}$ attributed to the original OMMT has shifted to $2.74^{\circ}$. According to Bragg's equation, the corresponding gallery space of the OMMT increased to 3.22 from $2.40 \mathrm{~nm}$ after graft polymerization. This result likely was caused by the intercalation of some PMMA chains into the interlayers of OMMT, leading to an increase in the gallery space. From the comparison of three XRD patterns, it could be seen that in the grafting copolymerization, the clay sheets of OMMT were intercalated by the continuous swelling of PMMA chains and finally uniformly dispersed in the organic PMMA formed. As an additional proof, the PMMA chains were grafted onto the substrate OMMT.

\subsection{Formation of radicals and preparation of OMMT- $g$-PMMA nanocomposite}

The formation of radicals and preparation of nanocomposite via the in situ graft copolymerization is shown in Figure 8. First, $\mathrm{Cu}^{3+}$ ion is a strong oxidizer in the alkaline aqueous solution [29]. $\mathrm{Cu}^{3+}$ ions readily reacted with some reactive groups including hydroxyl and amino groups on OMMT, resulting in the producing of the corresponding radical-cations, as well as $\mathrm{Cu}^{2+}$ ions. These radicalcations are chemically bound on the substrate OMMT. And then, the radical-cation further reacted with hydroxyl ion $\left(\mathrm{OH}^{-1}\right)$ provided by the alkaline medium so as to produce the macroradicals 


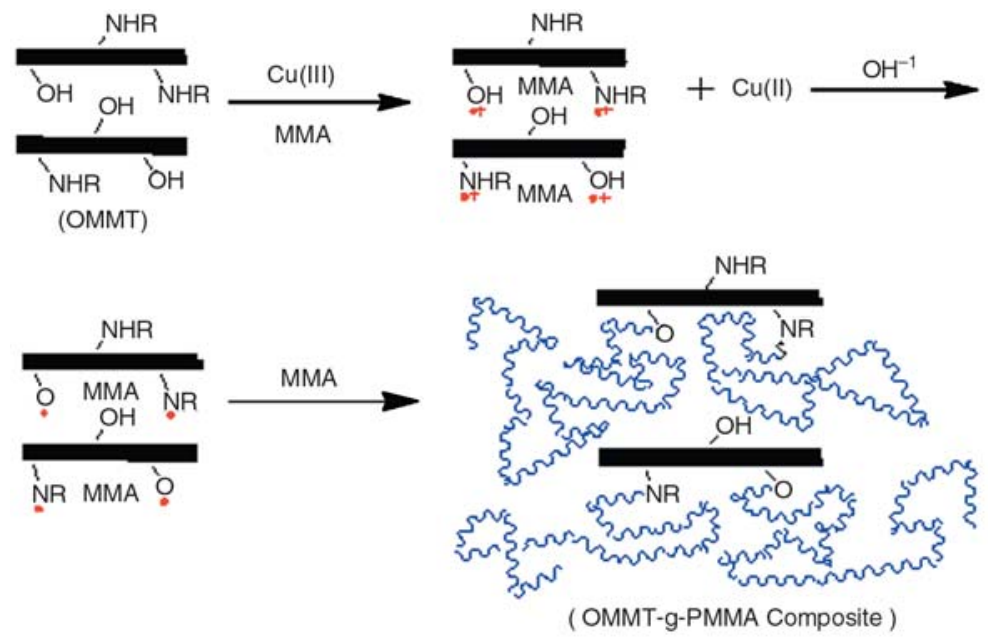

Figure 8. The proposed mechanism for the preparation for the nanocomposite by $\mathrm{Cu}^{3+}$

on OMMT. The macroradicals are sites for the subsequent grafting copolymerization of MMA onto OMMT. The monomers MMA in close vicinity to the macro-radicals were initiated and followed by the quick chain growth of PMMA in the interlayers of OMMT. The similar mechanism about the radical formation and initiation was also proposed by Savina et al. [30]. In fact, MMA and $\mathrm{Cu}^{3+}$ ions can diffuse into the interlayers of OMMT because of the usage of acetone/water mixture and surfactant used as modifier in OMMT. The interlayer structure of OMMT was intercalated due to the continuous swelling of the PMMA chains. Finally, the fine OMMT particles dispersed homogeneously in the PMMA formed, which was supported by the aforementioned XRD measurement. Because the radicals were generated via the redox reaction of $\mathrm{Cu}^{3+}$ with reactive groups in OMMT, nearly all of the PMMA chains were chemically bound to OMMT, not simply mixed. In conclusion, the composites of PMMA and OMMT formed simultaneously in course of the graft copolymerization of MMA onto OMMT.

\section{Conclusions}

The present article described a new initiator, potassium diperiodatocuprate $\left(\mathrm{Cu}^{3+}\right)$, for preparing the composite based on OMMT and PMMA through in situ polymerization. By investigating the effect of reaction conditions on the synthetic parameters, the optimum of synthetic procedure was given as follow: $\mathrm{pH}=11.6$; temperature $=25^{\circ} \mathrm{C} ; \mathrm{Cu}^{3+}$ concentration is $0.80 \cdot 10^{-3} \mathrm{~mol} / \mathrm{l}$; ratio of MMA to OMMT is fixed at 8 . From the experimental results, it could be seen that $\mathrm{Cu}^{3+}$ is an effective and practical initiator for the preparation of the organic/inorganic composite via the in suit polymerization. Moreover, the preparation of the intercalation-type composite could be conducted under milder conditions because of the lower activation energy in $\mathrm{Cu}^{3+}$ redox reaction. The grafting of PMMA chain onto OMMT and the improvement in the thermal stability were demonstrated by FTIR, TGA, and X-ray diffraction measurements. Further studies on the mechanical properties of OMMT- $g$-PMMA composite are now in progress.

\section{Acknowledgements}

The authors thank the Natural Science Foundation of Hebei Province (B2008000573) for the financial support. And, the authors also would like to thank the Institute of Chemistry, Chinese Academy of Sciences, Beijing, China, for the XRD measurement.

\section{References}

[1] Tang E. J., Cheng G. X., Ma X. L.: Preparation of nano-ZnO/PMMA composite particles via grafting of the copolymer onto the surface of zinc oxide nanoparticles. Powder Technology, 161, 209-214 (2006).

[2] Chow W. S., Mohd Ishak Z. A.: Mechanical, morphological and rheological properties of polyamide 6/ organo-montmorillonite nanocomposites. Express Polymer Letters, 1, 77-83 (2007).

[3] Avella M., Errico M. E., Gentile G.: PMMA based nanocomposites filled with modified $\mathrm{CaCO}_{3}$ nanoparticles. Macromolecular Symposia, 247, 140-146 (2007). 
[4] Qi R. R., Jin X., Zhou C. X.: Preparation and properties of polyethylene-clay nanocomposites by an in situ graft method. Journal of Applied Polymer Science, 102, 4921-4927 (2006).

[5] Zou H., Ma Q. Q., Tian Y., Wu S. S., Shen J.: Structure and properties of nanocomposites prepared by directly melt blending ethylene-co-vinylacetate and natural montmorillonite. Polymer Composites, 27, 529-532 (2006).

[6] Psarras G. C.: Hopping conductivity in polymer matrix-metal particles composites. Composites Part A: Applied Science and Manufacturing, 37, 15451553 (2006).

[7] Fan X. W., Lin L. J., Messersmith P. B.: Surface-initiated polymerization from $\mathrm{TiO}_{2}$ nanoparticle surfaces through a biomimetic initiator: A new route toward polymer-matrix nanocomposites. Composites Science and Technology, 66, 1198-1204 (2006).

[8] Chen Y., Qian Z., Zhang Z. C.: Novel preparation of magnetite/polystyrene composite particles via inverse emulsion polymerization. Colloids and Surfaces A: Physicochemical and Engineering Aspects, 312, 209-213 (2008).

[9] Kumar J., Singh R. K., Siwach P. K., Singh H. K., Singh R., Srivastava O. N.: Low field magneto-transport in LBSMO-PMMA composite. Journal of Magnetism and Magnetic Materials, 299, 155-160 (2006).

[10] Cai L. F., Mai Y. L., Rong M. Z., Ruan W. H., Zhang M. Q.: Interfacial effects in nano-silica/polypropylene composites fabricated by in-situ chemical blowing. Express Polymer Letters, 1, 2-7 (2007).

[11] Dhakal H. N., Zhang Z. Y., Richardson M. O. W.: Nanoindentation behaviour of layered silicate reinforced unsaturated polyester nanocomposites. Polymer Testing, 25, 846-852 (2006).

[12] Diagne M., Gueye M., Vidal L., Tidjani A.: Thermal stability and fire retardant performance of photo-oxidized nanocomposites of polypropylene-graft-maleic anhydride/clay. Polymer Degradation and Stability, 89, 418-426 (2005).

[13] Tidjani A., Wald O., Pohl M-M., Hentschel M. P., Schartel B.: Polypropylene-graft-maleic anhydridenanocomposites: I-Characterization and thermal stability of nanocomposites produced under nitrogen and in air. Polymer Degradation and Stability, 82, 133140 (2003)

[14] Rajendran S., Mahendran O., Kannan R.: Ionic conductivity studies in composite solid polymer electrolytes based on methylmethacrylate. Journal of Physics and Chemistry of Solids, 63, 303-307 (2002).

[15] Nah C. W., Ryu H. J., Kim W. D., Choi S-S.: Barrier property of clay/Acrylonitrile-butadiene copolymer nanocomposite. Polymers for Advanced Technologies, 13, 649-652 (2002).

[16] Zhang B., Li Y. F., Pan X. B., Jia X., Wang X. L.: Intercalation of acrylic acid and sodium acrylate into kaolinite and their in situ polymerization. Journal of Physics and Chemistry of Solids, 68, 135-142 (2007).
[17] Hiroyuki E., Michael M. L.: Synthesis of polymer/1T$\mathrm{TaS}_{2}$ layered nanocomposites. Materials Research Bulletin, 37, 1499-1507 (2002).

[18] Limpanart S., Khunthon S., Taepaiboon P., Supaphol P., Srikhirin T., Udomkichdecha W., Boontongkong Y.: Effect of the surfactant coverage on the preparation of polystyrene-clay nanocomposites prepared by melt intercalation. Materials Letters, 59, 2292-2295 (2005).

[19] Ballarin B., Facchini M., Dal Pozzo L., Martini C.: Comparison of different porous sol-gel matrices: template synthesis of polythiophene. Electrochemistry Communications, 5, 625-631 (2003).

[20] Luo W., Zhang W. A., Chen P., Fang Y. E.: Synthesis and properties of starch grafted poly[acrylamide-co(acrylic acid)]/montmorillonite nanosuperabsorbent via $\gamma$-ray irradiation technique. Journal of Applied Polymer Science, 96, 1341-1346 (2005).

[21] Rong M. Z., Zhang M. Q., Wang H. B., Zeng H. M.: Surface modification of magnetic metal nanoparticles through irradiation graft polymerization. Applied Surface Science, 200, 76-93 (2002).

[22] Hayashi S., Fujiki K., Tsubokawa N.: Grafting of hyperbranched polymers onto ultrafine silica: postgraft polymerization of vinyl monomers initiated by pendant initiating groups of polymer chains grafted onto the surface. Reactive and Functional Polymers, 46, 193-201 (2000).

[23] Tsubokawa N., Maruyama K., Sone Y., Shimomura M.: Graft polymerization of acrylamide from ultrafine silica particles by use of a redox system consisting of ceric ion and reducing groups on the surface. Polymer Journal, 21, 475-481 (1989).

[24] Liu Y. H., Liu Z. H., Zhang Y. Z., Deng K. L.: Graft copolymerizaztion of methyl acrylate onto chitosan initiated by potassium diperiodatocuprate (III). Journal of Applied Polymer Science, 89, 2283-2289 (2003).

[25] Liu Y. H., Liu X. H., Deng K. L., Liu Z. J.: Graft copolymerization of methyl acrylate onto poly(vinyl alcohol) initiated by potassium diperiodatonickelate(IV). Journal of Applied Polymer Science, 87, 529-534 (2003).

[26] Liu Y. H., Zhang Y. Z., Liu Z. H., Deng K. L.: Graft copolymerization of butyl acrylate onto casein initiated by potassium diperiodatonickelate(IV) in alkaline medium. European Polymer Journal, 38, 1619-1625 (2002).

[27] Liu Y. H., Bai L. B., Zhou W. Q., Liu Y. W., Li Y. X., Deng K. L.: Block copolymerization of poly(diethylene glycol phthalic anhydride) and methyl methacrylate initiated by potassium diperiodatonickelate(IV). Journal of Applied Polymer Science. 100, 1312-1317 (2006).

[28] Liu Y. H., Liu X. H., Liu Y. W., Zhang J. S., Deng K. L., Liu Z. J.: Graft copolymerization of methyl acrylate onto poly(vinyl alcohol) initiated by potassium diperiodatoargentate(III). Polymer International, 53, 1611-1616 (2004) 
[29] Timy P. J., Suresh M. T.: Oxidation of threonine by the analytical reagent diperiodatocuprate(III)-An autocatalysed reaction. Journal of Molecular Structure, 827, 137-144 (2007).

[30] Savina I. N., Mattiasson B., Galaev I. Y.: Graft polymerization of acrylic acid onto macroporous polyacrylamide gel (cryogel) initiated by potassium diperiodatocuprate. Polymer, 46, 9596-9603 (2005).
[31] Savina I. N., Galaev I. Y., Mattiasson B.: Anionexchange supermacroporous monolithic matrices with grafted polymer brushes of $\mathrm{N}, \mathrm{N}$-dimethylaminoethylmethacrylate. Journal of Chromatography A, 1092, 199-205 (2005).

[32] Chimatadar S. A., Basavaraj T., Thabaj K. A., Nandibewoor S. T.: Ruthenium(III) catalysed oxidation of gabapentin (neurontin) by diperiodatocuprate(III) in aqueous alkaline medium-A kinetic and mechanistic study. Journal of Molecular Catalysis A: Chemical, 267, 65-71 (2007). 